\title{
Floors number influence on the instability parameter of reinforced concrete wall- or core-braced buildings
}

\section{Influência do número de pavimentos no parâmetro de instabilidade de edifícios contraventados por paredes ou núcleos de concreto armado}

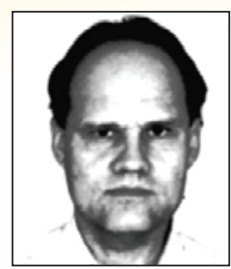

R. J. ELLWANGER a rjellwanger@pop.com.br

\begin{abstract}
This work aims to investigate the floors number influence on the instability parameter limit $\alpha$, of buildings braced by reinforced concrete walls and/ or cores. Initially, it is showed how the Beck and König discrete and continuous models are utilized in order to define when a second order analysis is needed. The treatment given to this subject by the Brazilian code for concrete structures design (NBR 6118) is also presented. It follows a detailed analytical study that led to the derivation of equations for the limit $\alpha_{1}$ as functions of the floors number; a series of examples is presented to check their accuracy. Results are analyzed, showing the precision degree achieved and topics for continuity of research in this field are indicated.
\end{abstract}

Keywords: instability, bracing structures, second order analysis.

\section{Resumo}

O presente trabalho tem por objetivo investigar a influência do número de pavimentos no limite $\alpha_{1}$ do parâmetro de instabilidade de edifícios contraventados por paredes e/ou núcleos de concreto armado. Inicialmente, é abordada a utilização dos modelos discreto e contínuo de Beck e König na definição da necessidade ou não de se realizar uma análise de segunda ordem; mostra-se também como esta questão é tratada pela norma de projeto de estruturas de concreto (NBR 6118). Na seqüência, apresenta-se um detalhado estudo analítico que levou ao estabelecimento de fórmulas para o limite $\alpha_{1}$ em função do número de andares, seguido de uma série de exemplos para testar a validade das mesmas. Os resultados são analisados, mostrando-se o grau de precisão obtido e indicando-se tópicos para a continuidade da pesquisa nesta área.

Palavras-chave: instabilidade, estruturas de contraventamento, análise segunda ordem.

\footnotetext{
Professor Associado, Departamento de Engenharia Civil, Universidade Federal do Rio Grande do Sul, e-mail: rjellwanger@pop.com.br, endereço
} postal: Rua Marcelo Gama 1189/401, CEP 90540-041, Porto Alegre-RS, Brasil. 


\section{Introduction}

\subsection{Second order effects and instability parameter}

When acting simultaneously on a building bracing structure with a certain flexibleness extent, gravity and wind loads may develop additional effects to those usually obtained in a first order linear analysis (in which the equilibrium is verified in the non deformed structure). They are the second order effects, in whose computation the material nonlinear behavior (physical nonlinearity) and the structure deflected shape (geometric nonlinearity) must be considered.

The work of Beck and König [1] provided an important contribution for the development of tall buildings global stability analysis theory. A simplified model for the bracing system of a building with equally spaced floors, shown in figure 1, was adopted. At first, all bracing substructures are grouped in a single column, while all braced elements (bearing elements that don't belong to the bracing system) are replaced by an assemblage of hinged bars, as shown in figure 1-a (discrete model). $W$ denotes the wind load applied on each floor, while $P$ and $V$ are the floor vertical loads, applied on the bracing substructures and braced elements, respectively. The loads $W$, $P$ and $V$ are considered with their characteristic values.

It can be proved that, when the system distorts laterally, the loads $V$ induce transmission of horizontal forces through the floor members to the bracing system, increasing its bending moments. It can also be proved that this increase is given by the sum of forces $V$ multiplied by the respective floors horizontal displacements. Therefore, in order to compute these bending moments including second order effects, the vertical loads acting on the bracing system are given by its proper $P$ loads added to the braced elements $V$ loads.

Thereafter, in order to make possible to analyze the whole building structure by means of a single differential equation, Beck and
König [1] adopted an equivalent approximate continuous system, shown in figure 1-b, with a continuous and uniform distribution of floors, vertical loads $(p=P / h$ and $v=V / h)$ and wind loads $(w=$ $W / h)$. The derivation of this equation originates a constant $\alpha$, as a function of the total vertical load, the height $H_{\text {tot }}$ and the bracing system horizontal stiffness $E l$. This constant is defined as the instability parameter, being expressed by:

$$
\alpha=H_{t o t} \sqrt{(p+v) H_{t o t} / E I}
$$

Beck and König [1] considered this single differential equation suitable for analysis of building structures with three or more floors. Furthermore, they concluded that second order effects may be neglected, provided that they don't represent an increase more than $10 \%$ on the first order effects. Studies done after the work of Beck and König [1], related by Vasconcelos [2] and Ellwanger [3], utilized this conclusion in order to establish a criterion defining if a second order analysis will be needed for a given bracing system. The Comité Euro-International du Béton recommendations (CEB [4]), an outstanding reference for this subject, preconized that the above mentioned criterion has to be applied comparing the global bending moment absolute values at the bracing system support

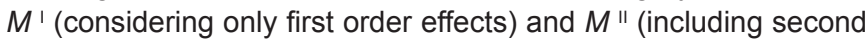
order effects), as stated below:

$$
M^{I I} \leq 1.1 M^{I}
$$

\section{Figure 1 - Bracing system simplified models}

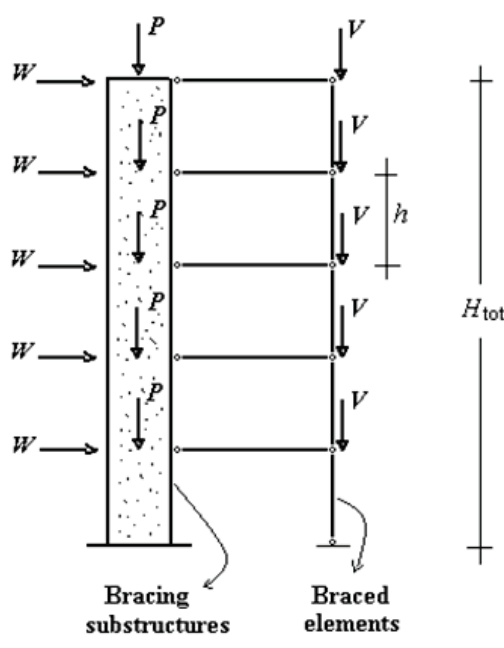

Discrete model
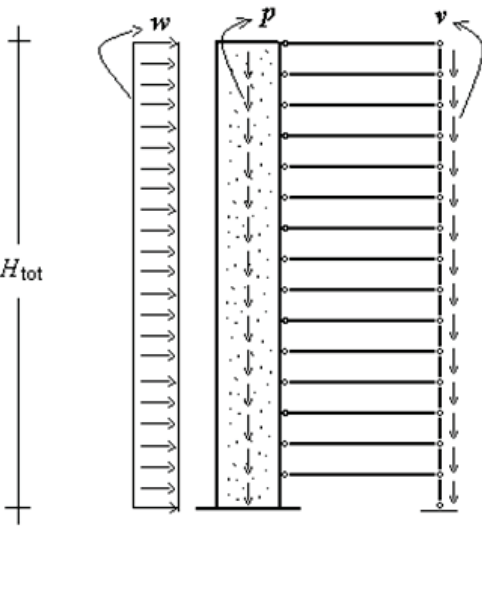

B Continuous model 
When $M^{\prime}$ and $M^{\text {" }}$ are expressed in function of the system loading and horizontal stiffness, the instability parameter $\alpha$, given by (1), becomes limited to particular values. The next section presents the treatment given to this subject by the present Brazilian code for concrete structures design (ABNT [5]).

Although not belonging to this work purpose, a mention deserves to be done to a computer aid method, based on the moment amplification factor $\gamma_{z}$. Presented in 1991 by Franco and Vasconcelos [6], it also applies the criterion of $10 \%$ increase in relation to first order effects, to define if a second order analysis is or not needed; however, in this case it is done for each combination of horizontal and vertical loads. Furthermore, under certain conditions, this method may itself constitute a second order analysis. These features caused this method to be rapidly disseminated and largely employed in buildings structures design. Nowadays, a great variety of powerful structural analysis programs is available, allowing an accurate modeling of building structures. Nevertheless, due to its simplicity, the method based on the instability parameter is frequently used in the preliminary design stages, especially in estimating the bracing system stiffness.

\subsection{ABNT NBR 6118 prescriptions}

The NBR 6118 code adopted the fundamental idea presented in [1] and [4], on determining in its section 15 that second order global effects are negligible when lower than $10 \%$ of the respective first order effects (fixed nodes structure). In order to "verify the possibility of dispensing the consideration of second order global efforts, in other words, to define if the structure may be classified as a fixed nodes one, without the need of a rigorous analysis", ABNT [5] presents two approximate procedures, respectively based on the instability parameter and the $\gamma_{z}$ factor. The first one just consists of the Beck and König [1] criterion application and determines that: "A symmetrical framed structure may be considered as a fixed nodes one, if its instability parameter $\alpha$ will be lesser than the value of $\alpha_{1}$, according to the expressions":

$$
\alpha=H_{\text {tot }} \sqrt{N_{\mathrm{k}} /\left(E_{\mathrm{CS}} I_{\mathrm{C}}\right)}
$$

$$
\alpha_{1}=0.2+0.1 n \quad \ni n \leq 3 \wedge \quad \alpha_{1}=0.6 \quad \text { э } n \geq 4
$$

" $n$ is the number of horizontal bars levels (floors) above the foundation or a slightly displaceable subsoil level. $H_{\text {tot }}$ is the structure total height, measured from the foundation top or from a slightly displaceable subsoil level. $N_{k}$ is the summation of all vertical loads acting on the structure (above the level considered for $H_{\text {tot }}$ computation), with their characteristic values. $E_{\mathrm{CS}} I_{\mathrm{C}}$ represents the summation of all columns stiffness values in the considered direction. $I_{C}$ is the moment of inertia considering the columns gross sections. $E_{C S}$ is the secant elasticity modulus, expressed by:"

$$
E_{C S}=0.85 E_{C i}=0.85 \times 5600 f_{c k}^{1 / 2}
$$

$E_{\mathrm{CS}}, \mathrm{E}_{\mathrm{Ci}}$ (tangent elasticity modulus) and $f_{\mathrm{ck}}$ (concrete compressive characteristic strength) are given in MPa. Furthermore, the NBR 6118 code determines different $\alpha_{1}$ values, depending on the bracing structure type: "The limit value $\alpha_{1}=0.6$, prescribed for $n \geq 4$, is generally applicable to building usual structures. It may be adopted for wall-columns assemblages and rigid frames associated to wall-columns. It may be increased until $\alpha_{1}=0.7$ in the case of bracing systems composed exclusively by wall-columns and must be reduced to $\alpha_{1}=0.5$ if there are only rigid frames."

In a second order analysis, the effects of both physical and geometric nonlinearities must be considered. In its item 15.7.3, ABNT [5] allows to consider the physical nonlinearity in an approximated manner. This is done by means of a reduction of the structural members stiffness factors $(E l)_{\mathrm{sec}}$ in function of $E_{\mathrm{Ci}} I_{\mathrm{C}}$, or of $E_{\mathrm{CS}} I_{\mathrm{C}}$ if equation (5) is used. Although the code restricts this procedure to four or more floors structures, in this work it will also be adopted for buildings with three or less floors. Therefore, this fact must be kept in mind when results of examples with few floors are analyzed. Thus, the columns reduced stiffness may be expressed by:

$$
(E I)_{\mathrm{sec}}=0.8 E_{C i} I_{C}=0.941 E_{C S} I_{C}
$$

\subsection{Reasons and targets of the research}

The NBR 6118 code represented an improvement in relation to the preceding one, on establishing procedures to verify the exemption of second order global effects consideration. Concerning to the instability parameter as a function of the floors number, it determines variable limits for buildings with less than four floors. However, the prescription of fixed limits $(0.5,0.6$ or 0.7 , depending on the bracing structure type) for a greater number of floors is questionable. For example, Ellwanger [3] found differences of about $12 \%$ between the limit coefficients $\alpha_{1}$ of a building braced exclusively by walls, with the number of floors varying from 5 until 30 . Considering that the instability parameter computation requires a square root extraction, the difference between the corresponding horizontal stiffness values reaches $25 \%$. Consequently, on verifying the exemption of performing a second order analysis, the error on determining the required horizontal stiffness can become significant.

This work aims to research a way of defining the instability parameter limit $\alpha_{1}$ for buildings braced by walls and/or cores, variable with the number of floors. At first, a computer aid method, based on the discrete model of Beck and König [1], is developed in order to determine the $\alpha_{1}$ limits for buildings with any number of floors. On applying this method, a series of $\alpha_{1}$ values is generated. Thereafter, the continuous model of Beck e König [1] is utilized in order to search approximated formulas that will reproduce this series of $\alpha_{1}$ values. The differential equations are solved by Galerkin method. The wind load is considered in two ways: constant along the building height and varying ac- 
Figure 2 - Bracing system with $\mathrm{n}$ floors

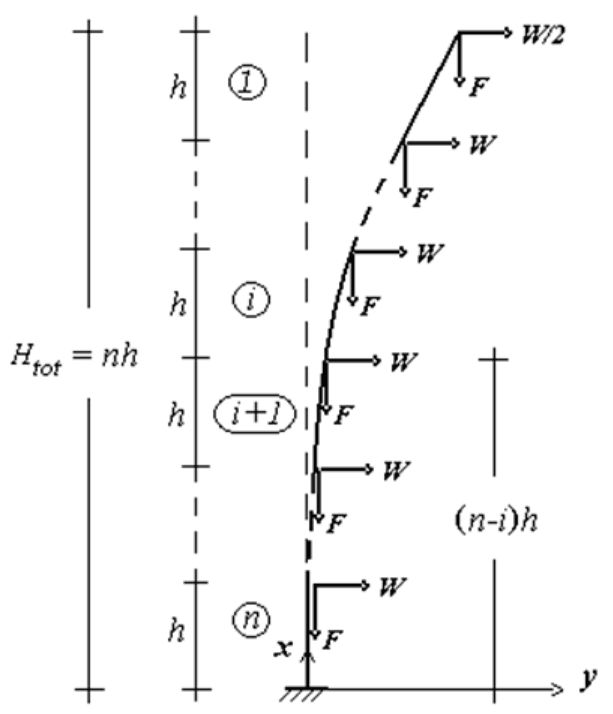

cording the prescriptions of NBR 6123 - Forces Due to Wind on Buildings (ABNT [7]). The deduced formulas are tested in 11 examples of buildings braced by walls and cores; 22 tests are performed, with the number of floors varying from 3 until 100 .

\section{Second order effects on the discrete model}

According to Beck and König [1] model, a bracing system composed by walls and/or cores may be modeled by a simple bar, behaving as a column. It has a high stiffness to shear, predominating flexural deflections. Figure 2 shows a cantilever bar of length $H_{\text {tot }}$, modeling the bracing system of a building with $n$ floors of the same height $h$. It is subject to gravity loads $F$ and wind loads $(W / 2$ at top and $W$ on the remaining floors). The loads are considered with their characteristic values.

Taking the bar deflections into account (geometric nonlinearity) and representing the material longitudinal elasticity modulus, the constant cross section moment of inertia and the functions of bending moments and horizontal displacements respectively by $E, J, M(x)$ and $y(x)$, it can be proved that the differential equation of motion and its respective solution for a generic bar interval $i$ are given by:

$$
\begin{aligned}
& M_{i}(x)=-E J \frac{d^{2} y_{i}}{d x^{2}}= \\
& -W\left\{\frac{n h-x}{2}+\sum_{j=1}^{i-1}[(n-j) h-x]\right\}+ \\
& F\left\{i y_{i}(x)-\sum_{j=0}^{i-1} y_{j+1}[(n-j) h]\right\}
\end{aligned}
$$

$$
\begin{aligned}
& y_{i}(x)=\frac{1}{i} \sum_{j=0}^{i-1} y_{j+1}[(n-j) h]+C_{2 i-1} \operatorname{sen}(\sqrt{i} a x) \\
& +C_{2 i} \cos (\sqrt{i} a x)+\frac{W}{i F}\left\{\frac{n h-x}{2}+\sum_{j=1}^{i-1}[(n-j) h-x]\right\}
\end{aligned}
$$

$C_{2 i-1}$ and $C_{2 i}$ are integration constants and the coefficient $a$ is expressed by:

$$
a^{2}=F / E J
$$

Bending moments inducing tension on the bar left side are considered negative. The subindexes attached to $M(x)$ and $y(x)$ indicate the validity interval of these functions. Applying equation (8) for the system top $(x=n h$ and $i=1)$, gives:

$$
C_{2}=-C_{1} \tan (n a h)
$$

Having a relation between $C_{1}$ and $C_{2}$ been obtained, it will now be shown how the integration constants concerning to a given bar interval can be expressed in function of the constants regarding to the preceding one. The function $\mathrm{y}_{i+1}(x)$ is obtained, replacing $i$ by $i+1$ in equation (8). Then, expressing successively $y_{i}(x)$ and $y_{i+1}(x)$ for $x=(n-i) h$ (transition between intervals $i$ and $i+1)$ and modifying these expressions adequately, results:

$$
\begin{gathered}
y_{i}[(n-i) h]-\frac{1}{i} \sum_{j=0}^{i-1} y_{j+1}[(n-j) h]-\frac{i W h}{2 F}= \\
C_{2 i-1} \operatorname{sen}[\sqrt{i}(n-i) a h]+C_{2 i} \cos [\sqrt{i}(n-i) a h]
\end{gathered}
$$

$$
\begin{gathered}
\frac{i}{i+1}\left\{y_{i+1}[(n-i) h]-\frac{1}{i} \sum_{j=0}^{i-1} y_{j+1}[(n-j) h]-\frac{i W h}{2 F}\right\}= \\
C_{2 i+1} \operatorname{sen}[\sqrt{i+1}(n-i) a h]+C_{2 i+2} \cos [\sqrt{i+1}(n-i) a h]
\end{gathered}
$$

The condition of equality between $y_{\mathrm{i}}(x)$ and $y_{\mathrm{i}+1}(x)$ for $x=(n-i)$ $h$ causes the left sides of equations (11) and (12) to be multiple among themselves. Consequently, (11) and (12) may be grouped into a single equation, as stated below:

$$
\begin{aligned}
& C_{2 i+1} \operatorname{sen}[\sqrt{i+1}(n-i) a h]+ \\
& C_{2 i+2} \cos [\sqrt{i+1}(n-i) a h]=B_{2}
\end{aligned}
$$


where

$B_{2}=\frac{i}{i+1}\left\{C_{2 i-1} \operatorname{sen}[\sqrt{i}(n-i) a h]+\right.$
$\left.C_{2 i} \cos [\sqrt{i}(n-i) a h]\right\}$

On the other hand, deriving equation (8) in relation to $x$ gives:

$$
\begin{gathered}
d y_{i} / d x=\sqrt{i} a\left[C_{2 i-1} \cos (\sqrt{i} a x)-\right. \\
\left.C_{2 i} \operatorname{sen}(\sqrt{i} a x)\right]-(W / i F)(i-1 / 2)
\end{gathered}
$$

Expressing equation (15) successively for intervals $i$ and $i+1$, leads to the rotation functions for these intervals. The condition of rotations continuity implies in equality between these functions for $x=(n-i) h$, resulting:

$$
\begin{aligned}
& C_{2 i+1} \cos [\sqrt{i+1}(n-i) a h]- \\
& C_{2 i+2} \operatorname{sen}[\sqrt{i+1}(n-i) a h]=B_{1}
\end{aligned}
$$

where

$$
\begin{gathered}
B_{1}=\frac{\sqrt{i}}{\sqrt{i+1}}\left\{C_{2 i-1} \cos [\sqrt{i}(n-i) a h]-\right. \\
\left.C_{2 i} \operatorname{sen}[\sqrt{i}(n-i) a h]\right\}+\frac{W}{2 i(i+1)^{3 / 2} a F}
\end{gathered}
$$

Modifying equations (13), (14), (16) and (17) adequately, $C_{2 i+1}$ and $C_{2 i+2}$ become expressed in function of $C_{2 i-1}$ and $C_{2 i}$, as follows:

\begin{tabular}{|l|}
\hline$C_{2 i+1}=B_{1} \cos [\sqrt{i+1}(n-i) a h]+$ \\
$B_{2} \operatorname{sen}[\sqrt{i+1}(n-i) a h]$ \\
\hline \hline $\begin{array}{l}C_{2 i+2}=B_{2} \cos [\sqrt{i+1}(n-i) a h]- \\
B_{1} \operatorname{sen}[\sqrt{i+1}(n-i) a h]\end{array}$ \\
\hline
\end{tabular}

Having a relation between the integration constants concerning to two successive bar intervals been determined, an expression for the bending moment on the bar support will now be deduced. The condition of null rotation at support is imposed, canceling equation (15) for $i=n$ (last interval) and $x=0$. Thereafter, $\mathrm{C}_{2 n-1}$ can be isolated, giving:

$$
C_{2 n-1}=(2 n-1) W / 2 n \sqrt{n} a F
$$

Deriving equation (15) in relation to $x$ and applying it for $i=n$, results:

$$
\begin{aligned}
& d^{2} y_{n} / d x^{2}=-n a^{2}\left[C_{2 n-1} \operatorname{sen}(\sqrt{n} a x)+\right. \\
& \left.C_{2 n} \cos (\sqrt{n} a x)\right]
\end{aligned}
$$

The expression for the support bending moment $M(0)$ is obtained, taking the first equality of equation (7) and making $i=n$ and $x=$ 0 . Then, $d^{2} y_{n} / d x^{2}$ given by (21), with $x=0$ and $a^{2}$ given by (9), is introduced, resulting:

$$
\begin{aligned}
& M(0)=M_{n}(0)=-E J d^{2} y_{n} / d x^{2}(0)= \\
& n a^{2} E J C_{2 n}=n F C_{2 n}
\end{aligned}
$$

The deduction of the $M(0)$ expression for buildings with a generic number $n$ of floors starts with the application of equation (10), so that $C_{2}$ results expressed in function of $C_{1}$. Thus, on applying equations (18) and (19) for the transition between the first and second intervals $(i=1)$, there will result expressions for $C_{3}$ and $C_{4}$ having $C_{1}$ as the only integration constant. The same will happen to the other constants, on applying those equations for the remaining intervals. Furthermore, due to the last parcel of the expression of $B_{1}$ given by (17), the successive applications of (18) and (19) generate expressions for the integration constants having a term multiplied by $W / a F$ that is independent of $C_{1}$. Thus, this procedure generates expressions for $C_{2 n-1}$ and $C_{2 n}$ (interval $n$ ) that may be put into the form:

$$
C_{2 n-1}=A_{1} C_{1}+D_{1} W / a F
$$

$$
C_{2 n}=A_{2} C_{1}+D_{2} W / a F
$$

The terms $\mathrm{A}_{1}, A_{2}, D_{1}$ and $D_{2}$ arise from the successive applications of equations (18) and (19). Combining equations (20), (22), (23) and (24), leads to the following expression for the bending moment at support:

$$
M(0)=\frac{n W}{a} \cdot \frac{A_{2}}{A_{1}}\left(\frac{2 n-1}{2 n \sqrt{n}}-D_{1}\right)+\frac{n W}{a} D_{2}
$$

On the other hand, the same bending moment, including only first order effects, is given by:

$$
M(0)=-W h\left(n / 2+\sum_{i=1}^{n-1} i\right)
$$


In order to verify the exemption of second order effects consideration, inequality (2) will be applied with the modules of $M^{\prime}$ and $M^{\prime \prime}$ respectively given by the $M(0)$ expressions of (26) and (25) (with changed signs, since these equations generate negative values for both $\mathrm{M}(0)$ ). On the other hand, according to the item 11.7.1 of NBR6118 code, the loads $W$ and $F$ must be multiplied by 1.4 and the coefficient $a$ by $\sqrt{1.4}$ (due to equation (9)), seeing that this criterion is applied for the ultimate state. Consequently:

$$
\begin{aligned}
& -\frac{n \times 1,4 W}{\sqrt{1,4} a} \cdot \frac{A_{2}}{A_{1}}\left(\frac{2 n-1}{2 n \sqrt{n}}-D_{1}\right)- \\
& \frac{n \times 1,4 W}{\sqrt{1,4} a} D_{2} \leq 1,1 \times 1,4 W h\left(n / 2+\sum_{i=1}^{n-1} i\right)
\end{aligned}
$$

Table 1 - Sequence of operations for the solution of inequality (28)

a) Input $n$ (number of floors).

b) Compute the right member of inequality (28).

c) Input the value (trial) of $\sqrt{1.4} \mathrm{ah}$.

d) Assign initial values: $\quad 1$ for $A_{1} ; \quad-\tan (\sqrt{1.4} n a h)$ for $A_{2} ; \quad 0$ for $D_{1}$ and $D_{2}$.

e) If $n=1$, go directly to step (f). If $\mathrm{n}>1$, s uccessively update the values of $A_{1}, A_{2}, D_{1}$ and $D_{2}$ for $n-1$ times, executing the set of operations presented below for intervals 2 to $n$ :

e.1) $\theta_{1}=\sqrt{i}(n-i) \sqrt{1.4} a h$

e.2) $B_{1}=\sqrt{i /(i+1)}\left(A_{1} \cos \theta_{1}-A_{2} \operatorname{sen} \theta_{1}\right)$

e.3) $B_{2}=[i /(i+1)]\left(A_{1} \operatorname{sen} \theta_{1}+A_{2} \cos \theta_{1}\right)$

e.4) $B_{W 1}=\sqrt{i /(i+1)}\left(D_{1} \cos \theta_{1}-D_{2} \operatorname{sen} \theta_{1}\right)+1 / 2 i(i+1)^{3 / 2}$

e.5) $B_{W 2}=[i /(i+1)]\left(D_{1} \operatorname{sen} \theta_{1}+D_{2} \cos \theta_{1}\right)$

e.6) $\theta_{2}=\sqrt{i+1}(n-i) \sqrt{1.4} a h$

e.7) $A_{1}=B_{1} \cos \theta_{2}+B_{2} \operatorname{sen} \theta_{2}$

e.8) $A_{2}=B_{2} \cos \theta_{2}-B_{1} \operatorname{sen} \theta_{2}$

e.9) $D_{1}=B_{W 1} \cos \theta_{2}+B_{W 2} \operatorname{sen} \theta_{2}$

e.10) $D_{2}=B_{W_{2}} \cos \theta_{2}-B_{W 1} \operatorname{sen} \theta_{2}$

f) Once $A_{1}, A_{2}, D_{1}$ and $D_{2}$ are determined, compute the left member of inequality (28).

g) Display the values of inequality (28) right and left members, with the purpose of comparison.

h) Inform decision about doing another trial; if it is affirmative, then go to (c), else terminate the procedure.

Remarks concerning to the expressions of step (e) operations:

a) They were deduced from equations (14), (17), (18) and (19), separating the parcels multiplied by $C_{1}$ from the ones multiplied by $W / \sqrt{1.4} a F$.

b) $i$ is the interval to be processed and the symbol "=", instead of mathematical equality, means "to assume the value of". 
It is implied that the terms $\mathrm{A}_{1}, A_{2}, D_{1}$ and $D_{2}$ of equations (23) and (24) will have been obtained, applying equations (14), (17), (18) and (19) with $\sqrt{1.4} a$ in place of $a$. Performing the required algebraic transformations, inequality (27) changes into:

$\frac{1}{\sqrt{1,4} a h} \cdot \frac{A_{2}}{A_{1}}\left(\frac{2 n-1}{2 \sqrt{n}}-n D_{1}\right)+$
$\frac{n D_{2}}{\sqrt{1,4} a h} \leq 1,1\left(n / 2+\sum_{i=1}^{n-1} i\right)$

For a small number of floors, it is feasible to derive expressions for $\mathrm{A}_{1}, A_{2}, D_{1}$ and $D_{2}$ as functions of $\sqrt{1.4}$ ah and then to replace them into the left member of (28). Thereafter, inequality (28) can be solved by trials, obtaining the factor $\sqrt{1.4} \mathrm{ah}$. However, for a greater number of floors, it is necessary to apply equations (18) and (19) many times, leading to very long expressions for $\mathrm{A}_{1}, A_{2}$ and consequently for the left member of (28). For buildings with more than four floors, this method of determination of $\sqrt{1.4}$ ah becomes impracticable. In face of this circumstance, an alternative method was developed in order to determine the factor $\sqrt{1.4}$ ah for buildings with a greater number of floors. Through this method, the solution is also obtained by means of trials. However, instead of deducing longer and longer expressions for $\mathrm{A}_{1}, A_{2}, D_{1}$ and $D_{2}$, successive trials are done, assigning an initial value to $\sqrt{1.4}$ ah and determining numerical values for those variables. In each trial, the abovementioned formulary is applied in such a way to obtain numerical values for the right and left members of inequality (28). When these values result close enough to be considered identical, then the factor $\sqrt{1.4}$ ah will have been determined.

Due to the great quantity of calculations, the method is computer aid. With the purpose of illustration, table 1 shows the sequence of operations for determining $\sqrt{1.4}$ ah by means of trials. Representing by $b$ the solution of inequality (28) obtained by this method and considering a as expressed by equation (9), it may be written:

$$
\sqrt{1.4} a h=\sqrt{1.4} \sqrt{F / E J} h \leq b
$$

It can be observed in figure 2 that $h=H_{t o t} / n$ and $F=N_{k} / n$, with $N_{k}$ as defined in subsection 1.2. On the other hand, since the wall or core behaves as a column, the physical nonlinearity may be considered, substituting $E J$ by $(E I)_{\text {sec }}$ given by (6). This changes inequality (29) into:

$$
H_{t o t} \sqrt{N_{k} / E_{C S} I_{C}} / n \sqrt{0.941 n} \leq b / \sqrt{1.4}
$$

On comparing (30) with equations (3) and (4), it is concluded that the limit $\alpha_{1}$ of the instability parameter may be expressed by:

$$
\alpha_{1}=b n \sqrt{0.941 n} / \sqrt{1.4}=0.82 b n^{3 / 2}
$$

Therefore, once the desired number of floors $(n)$ is introduced into the sequence of operations of table 1 , the value of $b$ can be determined; then, the value of the limit coefficient $\alpha_{1}$ can be obtained, applying equation (31). This was done for a series of floors quantities and the

\begin{tabular}{|c|c|c|c|c|c|}
\hline $\mathbf{n}$ & $\alpha 1$ & $\alpha 1$ (2) & $\mathrm{n}$ & $\alpha 1$ & $\alpha \mathbf{l}$ (2) \\
\hline 1 & 0.425 & 0.426 & 25 & 0.754 & 0.754 \\
\hline 2 & 0.571 & 0.573 & 30 & 0.757 & 0.757 \\
\hline 3 & 0.631 & 0.631 & 35 & 0.759 & 0.759 \\
\hline 4 & 0.663 & 0.663 & 40 & 0.761 & 0.761 \\
\hline 5 & 0.683 & 0.683 & 50 & 0.763 & 0.763 \\
\hline 6 & 0.697 & 0.697 & 60 & 0.765 & 0.765 \\
\hline 7 & 0.707 & 0.707 & 70 & 0.766 & 0.766 \\
\hline 8 & 0.715 & 0.715 & 80 & 0.767 & 0.767 \\
\hline 9 & 0.721 & 0.721 & 100 & 0.768 & 0.768 \\
\hline 10 & 0.726 & 0.726 & 125 & 0.769 & 0.769 \\
\hline 12 & 0.734 & 0.733 & 165 & 0.770 & 0.770 \\
\hline 14 & 0.739 & 0.739 & 250 & 0.771 & 0.771 \\
\hline 16 & 0.743 & 0.743 & 500 & 0.772 & 0.772 \\
\hline 18 & 0.746 & 0.746 & $\geq 1100$ & 0.773 & 0.773 \\
\hline 20 & 0.749 & 0.749 & - & - & - \\
\hline
\end{tabular}
results are displayed in the second and fifth columns of table 2.

Table 2 - Values of $\alpha 1$ in function of the floors number (uniform wind load) 


\section{The continuous model and the derivation of an approximated formula for $\alpha_{1}$}

The values of $\alpha_{1}$, presented in table 2, may be considered as "exact solutions" (in the context of the discrete model of figure 2) for the instability parameter limit of bracing systems composed exclusively by walls and/or cores. Nevertheless, a question remains unsolved, since the method developed in the preceding section doesn't provide an explicit formula for obtaining the limit coefficient $\alpha_{1}$. Thus, the aim of this section is to deduce approximated formulas that give the value of $\alpha_{1}$ in function of the number of floors with an adequate accuracy. At first, the case of uniform wind load is considered; then, the case of wind load distributed according to NBR 6123 [7] prescriptions is treated.

It can be verified that the continuous model, shown in figure 1-b, is inadequate for buildings with few floors. This is primarily because the simulation of a concentrated loads set by means of a distributed load only provides a good accuracy if the number of concentrated loads is large. In buildings with few floors, the model of figure 1-b fails mainly by no capturing the effect of the top vertical load. In order to avoid this drawback, the continuous model of figure 3 , with a vertical load $P$ concentrated at the building top, is adopted.

\subsection{Uniformly distributed wind load}

If the distortions effect is neglected in the model of figure 3-a, it can be proved that the linear solution, in terms of the rotations $\phi(x)$, for a uniform wind load of rate $w$, is given by:

$$
\phi(x)=\frac{w \ell^{3}}{6 E J}\left[1-\left(1-\frac{x}{\ell}\right)^{3}\right]
$$

Now, considering the distortions effect, the bending moments function will be expressed by:

$$
\begin{aligned}
& M(x)=-w(\ell-x)^{2} / 2-P[y(\ell)-y(x)]- \\
& \int_{x}^{\ell} q[y(\xi)-y(x)] d \xi
\end{aligned}
$$

Representing by $Y(x)$ the primitive function of the horizontal displacements $y(x)$, equation (33) changes into:

$$
\begin{aligned}
& M(x)=-w(\ell-x)^{2} / 2-P[y(\ell)-y(x)] \\
& -q[Y(\ell)-Y(x)-(\ell-x) y(x)]
\end{aligned}
$$

Equating $-E J d^{2} y / d x^{2}$ to $M(x)$ given by (34), leads to the differential equation of motion. Deriving it in relation to $x$, remembering that $d y /$ $d x=\phi(x)$ and re-arranging, results:

$$
E J d^{2} \phi / d x^{2}+[P+q(\ell-x)] \phi(x)+w(\ell-x)=0
$$

In order to solve equation (35), Galerkin method will be adopted. It consists in obtaining an approximated solution of the form:

$$
\phi(x) \cong \bar{\phi}(x)=\sum_{i=1}^{m} a_{i} \varphi_{i}(x)
$$

where $\varphi_{\mathrm{i}}(x)(i=1,2, \ldots, m)$ are previously chosen functions

Figure 3 - Continuous model for the bracing system

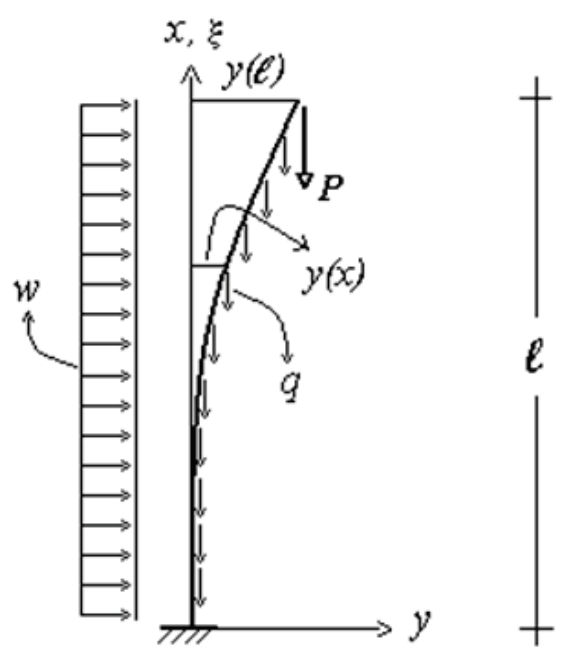

Uniform wind load
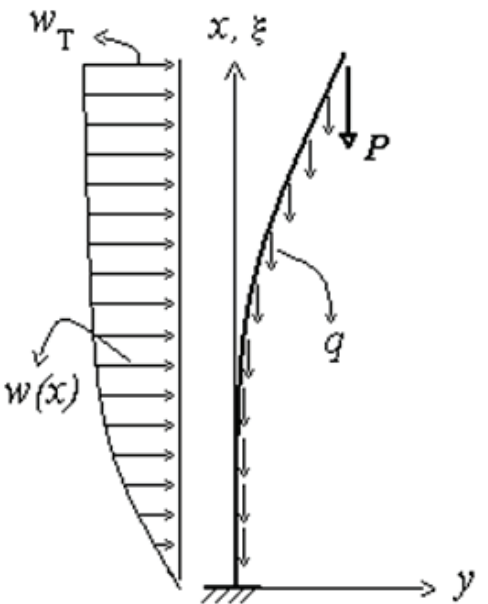

B Wind load according to NBR 6123 prescriptions 
and the $a_{\mathrm{i}}$ are coefficients to be determined. More detailed considerations about Galerkin method can be seen in Kantorovitch and Krylov [8]. On applying the method for the actual case, the summation of equation (36) will be reduced to a single parcel $(m=1)$ and a function proportional to the linear solution, given by (32), will be adopted for $\varphi_{1}(x)$. Consequently, equation (36) changes into:

$$
\phi(x) \cong a_{1} \varphi_{1}(x)=a_{1}\left[1-\left(1-\frac{x}{\ell}\right)^{3}\right]
$$

In order to obtain $\alpha_{1}$, the following equation must be solved:

$$
\begin{aligned}
& \int_{0}^{\ell}\left\{-\frac{6 E J}{\ell^{2}} a_{1}\left(1-\frac{x}{\ell}\right)+[P+q(\ell-x)] .\right. \\
& \left.a_{1}\left[1-\left(1-\frac{x}{\ell}\right)^{3}\right]+w(\ell-x)\right\} \varphi_{1}(x) d x=0
\end{aligned}
$$

The term multiplied by $\varphi_{1}(x)$ is the differential operator regarding to equation (35). Thus, introducing $\varphi_{1}(x)$ given by (37), performing the integration and isolating $\alpha_{1}$, gives:

$$
a_{1}=\frac{w \ell^{3}}{6 E J-(15 / 7) P \ell^{2}-(3 / 4) q \ell^{3}}
$$

Replacing (39) into (37), leads to the function $\phi(x)$. Comparing it with (32), it can be observed that the geometric nonlinearity arises through the terms that are subtracted from $6 E J$ in the denominator of $\alpha_{1}$. Integrating $\phi(x)$ twice, leads successively to the functions $y(x)$ and $Y(x)$. Substituting them into equation (34) and applying it for $x=0$, leads to the following expression for the bending moment at support:

$$
M(0)=-\frac{w \ell^{2}}{2}-\frac{7}{5} \cdot \frac{w \ell^{2}\left(5 P \ell^{2}+2 q \ell^{3}\right)}{56 E J-20 P \ell^{2}-7 q \ell^{3}}
$$

In order to verify the exemption of second order effects consideration, inequality (2) will be applied, replacing $M$ " by the modulus of $M(0)$ given by (40), with the loads multiplied by 1.4. Hence:

$$
\begin{aligned}
& 1.4 \frac{w \ell^{2}}{2}+\frac{7}{5} \quad \frac{1.4 w \ell^{2} \times 1.4\left(5 P \ell^{2}+2 q \ell^{3}\right)}{56 E J-20 \times 1.4 P \ell^{2}-7 \times 1.4 q \ell^{3}} \leq \\
& 1.1 \times 1.4 \frac{w \ell^{2}}{2}
\end{aligned}
$$

Performing successive algebraic transformations, equation (41) changes into:

$(8 P+3.15 q \ell) \ell^{2} / E J \leq 2$

The physical nonlinearity may be considered, substituting EJ by $(E I)_{\mathrm{sec}}$ given by (6). Then, extracting the square root of equation (42) both sides and re-arranging, gives:

$$
\ell \sqrt{(2.54 P+q \ell) / E_{C S} I_{C}} \leq 0.773
$$

It must be remembered that the aim of this section is to obtain a formula for $\alpha_{1}$ in function of the number of floors. In order to do it starting from equation (43), it is necessary to find a relation between $q \ell$ and $P$ in function of the number of floors, in such a way that the application of (43) provides $\alpha_{1}$ values as close to the ones presented in the second and fifth columns of table 2 as possible. On the other hand, representing $H_{\text {tot }}$ by $\ell$, equation (3) combined with (4) may be expressed as follows:

$\ell \sqrt{N_{k} / E_{C S} I_{C}} \leq \alpha_{1}$

The factor $E_{C S} I_{C}$ can be isolated in both equations (43) and (44). Then, equating the resulting expressions one another and considering that the total vertical load $N_{\mathrm{k}}$ is given by the sum of $P$ and $q \ell$, it can be proved that:

$$
\frac{q \ell}{P}=\frac{2.54 \alpha_{1}^{2}-0.773^{2}}{0.773^{2}-\alpha_{1}^{2}}
$$

The next step is to input the series of $\alpha$ values presented in table 2 (discrete model) into equation (45), determining successive values for $q \ell / P$ corresponding to successive floors quantities and listing them into table 3 . Thereafter, an equation that provides values for $q \ell / P$ in function of $n$ with an adequate accuracy must be searched. A possible solution consists of the following straight line equation:

$$
q \ell / P=1.201 n-1.528
$$

Finally, considering that $\ell$ is the height $H_{\text {tot }}$ and combining equations (44), (45) and (46), results:

$$
\begin{aligned}
& H_{\text {tot }} \sqrt{N_{k} / E_{C S} I_{C}} \leq \alpha_{1}= \\
& 0.773 \sqrt{(n-0.44) /(n+0.84)}
\end{aligned}
$$




\section{Table 3 - Values of $\mathrm{q} / \mathrm{P}$ in function of the floors number}

\begin{tabular}{|cccc|}
$\begin{array}{c}\text { Number } \\
\text { of floors }\end{array}$ & qR/P & $\begin{array}{c}\text { Number } \\
\text { of floors }\end{array}$ & qR/P \\
\hline 1 & -0.333 & 20 & 22.6 \\
2 & 0.849 & 25 & 29.2 \\
3 & 2.07 & 30 & 35.0 \\
4 & 3.28 & 35 & 40.4 \\
5 & 4.48 & 40 & 47.3 \\
6 & 5.69 & 50 & 57.2 \\
\hline 7 & 6.88 & 60 & 72.0 \\
8 & 8.12 & 70 & 82.5 \\
9 & 9.30 & 80 & 97.0 \\
10 & 10.5 & 100 & 116 \\
\hline 12 & 13.1 & 125 & 147 \\
14 & 15.3 & 165 & 196 \\
16 & 17.7 & 250 & 291 \\
18 & 19.9 & 500 & 593 \\
\hline
\end{tabular}

In this way, an expression for $\alpha_{1}$ in function of the floors number was obtained. Equation (47) was applied for the same series of floors numbers of table 2 and the resulting values are presented in its third and sixth columns. A quasi perfect coincidence between the values of $\alpha_{1}$ regarding to the discrete and continuous models can be verified; the respective graphs, shown in figure 4 for buildings ranging from 1 to 30 floors, appear superposed.

\subsection{Wind load distributed according to NBR 6123 prescriptions}

Figure 3-b presents the model of a bracing system subject to a wind load of rate $w(x)$, variable along the height, reaching a value $w_{T}=w(\ell)$ on the building top. According to NBR 6123 (ABNT [7]) prescriptions, the rate $w(x)$ may be expressed as follows:

$$
w(x)=K(x / 10)^{p}
$$

$\mathrm{K}$ is a constant that depends on many factors, as: surface of the building face perpendicular to the wind direction; relation between the building dimensions; basic wind speed and topographic, statistical and gust factors, as defined by NBR 6123. The exponent $p$ is the double of the meteorological parameter $p$, varying from 0.06 until 0.175 and depending on the building dimensions and ground roughness. Thus, $p$ can vary from 0.12 until 0.35 .

It can be proved that the rotations $\phi(x)$ and the support bending moment $M(0)$, due to first order effects exclusively, are respectively given by:

$$
\begin{aligned}
& \phi(x)=\frac{w_{T}}{E J} . \\
& \frac{(p+1) \ell^{2} x-(p+2) \ell x^{2} / 2+x^{p+3} /(p+3) \ell^{p}}{(p+1)(p+2)}
\end{aligned}
$$

$$
M(0)=-w_{T} \ell^{2} /(p+2)
$$

On the other hand, considering the distortions effect and following the same deductive sequence that led to equations (34) and (35), it can be proved that the bending moments function and the differential equation of motion are respectively given by:

$$
\begin{gathered}
M(x)=-w_{T} \cdot \frac{(p+1) \ell^{2}-(p+2) \ell x+\frac{x^{p+2}}{\ell^{p}}}{(p+1)(p+2)}- \\
P[y(\ell)-y(x)]-q[Y(\ell)-Y(x)+(\ell-x) y(x)]
\end{gathered}
$$

$$
\begin{aligned}
& E J \frac{d^{2} \phi}{d x^{2}}+[P+q(\ell-x)] \phi(x)+ \\
& w_{T} \cdot \frac{\ell-x^{p+1} / \ell^{p}}{p+1}=0
\end{aligned}
$$

In order to solve equation (52), Galerkin method will be used, adopting a function $\varphi_{1}(x)$ proportional to the linear solution given by (49). In the derivation proceeding, due to the great extension of the expressions that are generated, the formulary will be particularized for $p=0.35$, which corresponds to a meteorological parameter $\bar{p}$ of 0.175 , regarding to a terrain with category $\mathrm{V}$ roughness. This value of $p$ results in the most far from uniform distribution loading pattern. Thus, following the same deductive sequence that led to equation (40), gives an expression for the

\section{Figure 4 - Graphs $\alpha 1$ x number of floors}

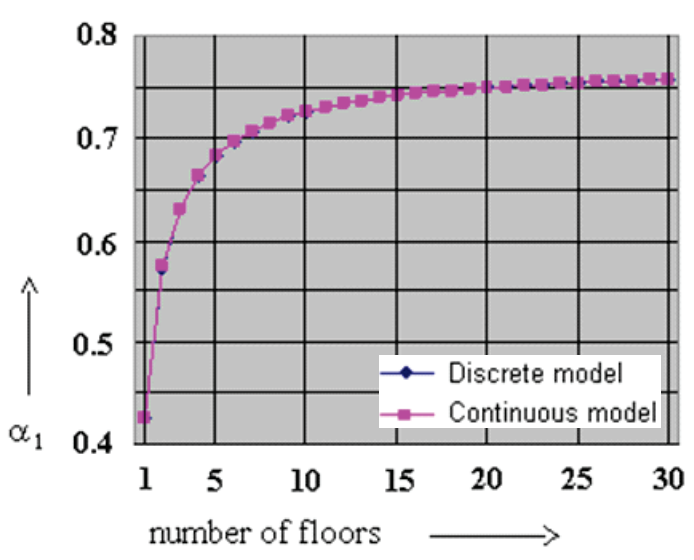


support bending moment including second order effects, as stated below:

$M(0)=-0.4255 w_{T} \ell^{2}-$
$\frac{0.3505 w_{T} \ell^{2}\left(2.516 P \ell^{2}+q \ell^{3}\right)}{7.95 E J-2.886 P \ell^{2}-q \ell^{3}}$

In order to verify the exemption of second order effects consideration, the moments $M^{\prime}$ and $M^{\prime \prime}$ will be replaced by the modules of $M(0)$ respectively given by (50) and (53), with the loads multiplied by 1.4 . In this way, applying inequality (2), replacing $E J$ by $(E l)_{\mathrm{sec}}$ given by (6) (physical nonlinearity) and performing the required algebraic transformations, results:

$\ell \sqrt{(2.556 P+q \ell) / E_{C S} I_{C}} \leq 0.7606$

Due to the similarity between equations (54) and (43), the same $\alpha_{1}$ variation pattern of uniform wind load will be assumed, with the coefficient 0.773 of (43) being changed to 0.7606 . Consequently, an expression for $\alpha_{1}$ in function of the floors number, similar to equation (47), is obtained for wind load distributed according to NBR 6123 prescriptions, as follows:

$H_{\text {tot }} \sqrt{N_{k} / E_{C S} I_{C}} \leq \alpha_{1}=$
$0.7606 \sqrt{(n-0.44) /(n+0.84)}$

Isolating $I_{C}$ in equation (55), gives:

$$
I_{C} \geq 1.729 \times \frac{n+0.84}{n-0.44} \times \frac{N_{k} H_{t o t}^{2}}{E_{C S}}
$$

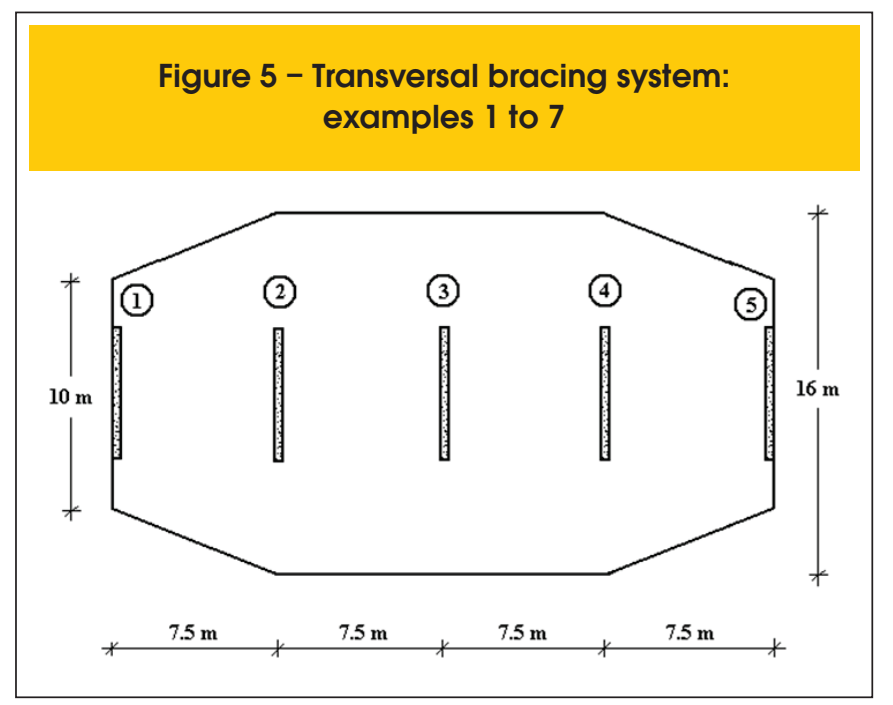

Equation (56) is very useful in the preliminary stage of a bracing system design, especially when the aim is to obtain a fixed nodes structure, according to NBR 6118 definition.

\section{Examples}

\subsection{Description of the tests}

The plan of figure 5 shows the basic configuration of the transversal bracing system of a building with an oblong octagonal shape on plane, being composed by walls 1 to 5 . This system was employed in buildings having $3,5,10,15,20,30$ and 40 floors, constituting examples 1 to 7 . In the same way, figure 6 shows the basic configuration of the transversal bracing system composed by walls 1 to 7 , which was employed in buildings with $50,60,80$ and 100 floors, constituting examples 8 to 11 . In examples 10 and 11, channel-shaped cores, indicated by the broken lines of figure 6 , were utilized in place of walls 1 and 7 .

In all the examples, it was adopted a story-height of $3 \mathrm{~m}$, as well as a concrete with $f_{\text {ck }}=25 \mathrm{MPa}$, resulting in an elasticity modulus $E_{\mathrm{CS}}=23800 \mathrm{MPa}$. A total vertical load of $10 \mathrm{kN} / \mathrm{m}^{2}$ per floor (characteristic value) was considered. A wind pressure of $1.5 \mathrm{kN} /$ $\mathrm{m}^{2}$ (characteristic value), constant along the height, was initially adopted, since it was an experience with a formulation based on a model with constant wind load.

Each of the 11 bracing systems was tested, aiming to determine the relation between vertical loads and horizontal stiffness that would result in a $10 \%$ increase on the global moment at building support, concerning to first order analysis; in this way, the limit $\alpha_{1}$ for the instability parameter was determined. The procedure applied in each test consisted in assigning initial dimensions to the walls cross sections and performing a second order analysis, employing the P-Delta method with double precision processing. More detailed considerations about P-Delta method can be seen in Smith and Coull [9]. After, this second order analysis was successively repeated, adjusting the cross sections dimensions until achieve the desired $10 \%$ increase on the support global moment. The physical nonlinearity was

\section{Figure 6 - Transversal bracing system:} examples 8 to 11

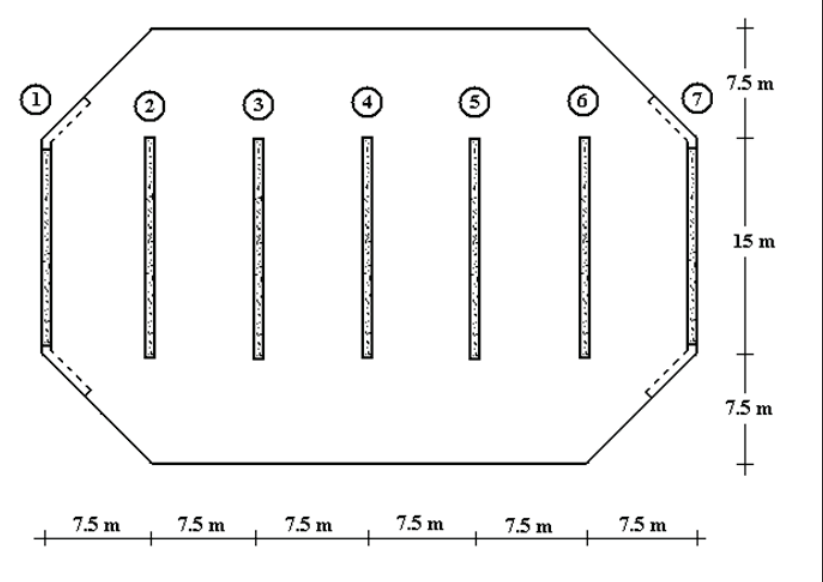


Table 4 - Results for uniform wind load

\begin{tabular}{|c|c|c|c|c|c|c|}
\hline Example & $\mathbf{n}$ & $\alpha 1(1)$ & $\alpha 1(2)$ & $I_{c}\left(m^{4}\right)$ & Walls & Cross section dimensions (cm) \\
\hline 1 & 3 & 0.631 & 0.632 & 0.1226 & 5 & $20 \times 114$ \\
\hline 2 & 5 & 0.683 & 0.684 & 0.4852 & 5 & $20 \times 180$ \\
\hline 3 & 10 & 0.726 & 0.726 & 3.441 & 5 & $20 \times 346$ \\
\hline 4 & 15 & 0.741 & 0.741 & 11.15 & 5 & $30 \times 447$ \\
\hline 5 & 20 & 0.749 & 0.749 & 25.88 & 5 & $30 \times 592$ \\
\hline 6 & 30 & 0.757 & 0.757 & 85.53 & 5 & $40 \times 800$ \\
\hline 7 & 40 & 0.761 & 0.761 & 200.7 & 5 & $48 \times 1000$ \\
\hline 8 & 50 & 0.763 & 0.763 & 1004 & 7 & $51 \times 1500$ \\
\hline 9 & 60 & 0.765 & 0.765 & 1727 & 5 & $60 \times 1771$ \\
\hline- & - & - & - & - & +2 & $60 \times 1500$ \\
\hline 10 & 80 & 0.767 & 0.767 & 4073 & 5 & $80 \times 2000$ \\
\hline- & - & - & - & - & +2 & $85 \times 1500$ (web) and $85 \times 354$ (edges) \\
\hline 11 & 100 & 0.768 & 0.768 & 7924 & 5 & $120 \times 2200$ \\
\hline- & - & - & - & - & +2 & $120 \times 1500$ (web) and $120 \times 472$ (edges) \\
\hline
\end{tabular}

considered by means of the individual bars stiffness reduction, expressed by equation (6). Due to the bracing double symmetry in plane, the analyses were performed using a plane frame model, with the walls joined among themselves by hinges representing the floor slabs.

Subsequently, the 11 examples were re-analyzed, considering a along the height variable wind load. The NBR 6123 prescriptions were observed, considering the following parameters: basic wind speed of $45 \mathrm{~m} / \mathrm{s}$; topographic and statistical factors equal to 1.0; and ground roughness of category $\mathrm{V}$, corresponding to big cities centers. In order to estimate the initial dimensions of the walls cross sections, equation (56) was applied.

\subsection{Results discussion}

The fourth column of table 4 shows the values of $\alpha_{1}$ found in the 11 examples, taking an uniform wind load into account. The following columns contain the values of the total gross inertia $I_{C}$ and the corresponding number of walls and cross section dimensions that led to the just mentioned values of $\alpha_{1}$. It can be observed that it

Table 5 - Results for wind load according to NBR 6123 prescriptions

\begin{tabular}{|c|c|c|c|c|c|c|c|}
\hline Example & $\mathbf{n}$ & $\alpha 1(3)$ & $I_{c, 1}\left(m^{4}\right)$ & $\alpha 1(4)$ & $I_{c, 2}\left(m^{4}\right)$ & Walls & Cross section dimensions (cm) \\
\hline 1 & 3 & 0.621 & 0.1271 & 0.625 & 0.1256 & 5 & $20 \times 115$ \\
\hline 2 & 5 & 0.672 & 0.5024 & 0.674 & 0.4989 & 5 & $20 \times 182$ \\
\hline 3 & 10 & 0.714 & 3.559 & 0.716 & 3.544 & 5 & $20 \times 349$ \\
\hline 4 & 15 & 0.729 & 11.52 & 0.730 & 11.48 & 5 & $30 \times 451$ \\
\hline 5 & 20 & 0.737 & 26.75 & 0.737 & 26.73 & 5 & $30 \times 598$ \\
\hline 6 & 30 & 0.745 & 88.40 & 0.745 & 88.36 & 5 & $40 \times 809$ \\
\hline 7 & 40 & 0.749 & 207.4 & 0.749 & 207.3 & 5 & $50 \times 1000$ \\
\hline 8 & 50 & 0.751 & 1038 & 0.751 & 1037 & 7 & $53 \times 1500$ \\
\hline 9 & 60 & 0.753 & 1785 & 0.753 & 1785 & 5 & $60 \times 1800$ \\
\hline - & - & - & - & - & - & +2 & $60 \times 1500$ \\
\hline 10 & 80 & 0.755 & 4209 & 0.755 & 4203 & 5 & $84 \times 2000$ \\
\hline - & - & - & - & - & - & +2 & $85 \times 1500$ (web) and $85 \times 354$ (edges) \\
\hline 11 & 100 & 0.756 & 8195 & 0.757 & 8191 & 5 & $120 \times 2200$ \\
\hline- & - & - & - & - & - & +2 & $25 \times 1500$ (web) and $125 \times 500$ (edges) \\
\hline
\end{tabular}


was necessary to replace the plane walls 1 and 7 of examples 10 and 11 by channel-shaped cores. Furthermore, the $\alpha_{1}$ values obtained from equation (47) are included in the third column. A nearly complete coincidence between them and the values found in the examples can be noticed, covering three significant digits, except for examples 1 and 2 in which, nevertheless, the differences are lower than $0.2 \%$.

Table 5 practically has the same arrangement of table 4 , showing results for wind load distributed according to NBR 6123 prescriptions. It has an additional column presenting the values initially assigned for the total gross inertia $I_{C}$, resulting from application of equation (56). It can be observed that these values, as well as the $\alpha_{1}$ values predicted by equation (55) are in good agreement with those obtained in the examples. A coincidence covering three significant digits of $\alpha_{1}$ is verified in examples 5 to 10 , while differences lower than $0.7 \%$ occur in the remaining ones.

The values shown on table 5 fifth column denote that the limits $\alpha_{1}=0.5$ and $\alpha_{1}=0.7$, respectively prescribed by the NBR 6118 code for buildings with 3 and with more than 10 floors, are conservative. However, the contrary happens to buildings with a floors number between 4 and 7 . Interpolations done with the $\alpha_{1}$ values regarding to examples 1 to 3 led to values lower than the limit 0.7 , prescribed for those floors quantities.

\section{Conclusions}

The limit values $\alpha_{1}$ of the instability parameter obtained for wind load distributed according to NBR 6123 prescriptions, presented in table 5 , vary from a minimum of 0.625 in example 1 until a maximum of 0.757 in example 11 . The proportion between these extreme values is slightly higher than 1.2:1. Since their computation includes a square root extraction, the proportion between the radicands (vertical load/horizontal stiffness relations) associated to these extreme values is close to 1.5:1. The extent of this variability shows the importance of having a way of predicting a limit $\alpha_{1}$ appropriated to the floors number of a given building to be designed, in place of the fixed values prescribed by the NBR 6118 code. For example, regarding to the fixed value 0.7 , equation (55) gives values ranging from 0.65 (4 floors) until 0.76 (90 or more floors).

In this work, a method based on the Beck and König [1] discrete model (Figure 1-a), considering equally spaced floors and uniform wind load, was initially developed. The method consists in solving inequality (28) by means of trials and then to input its solution into equation (31), obtaining $\alpha_{1}$. Since the method is computer aid, $\alpha_{1}$ can be obtained for any number of floors, as can be seen in table 2. Thereafter, these results were employed in the following phase of this study, in which a variant of the continuous model of Beck and König [1] (Figure 3) was adopted, aiming to deduce approximated formulas for $\alpha_{1}$ in function of the floors number; in this way, equations (47) and (55) became determined. The results obtained in the examples revealed a good accuracy of these equations, respectively for the cases of uniform wind load and wind load distributed according to NBR 6123 prescriptions for roughness category $\checkmark$ terrain.

It can be verified that the values of $\alpha_{1}$ found for these loading patterns, mentioned on tables 4 and 5 , are quite close among themselves, with differences lower than $1.7 \%$. This proves that the Beck and König [1] model, on considering a uniformly distributed wind load, incurs in an irrelevant error. The graph of figure 7 illustrates

\section{Figure 7 - Values of $\alpha 1$ obtained in the examples}

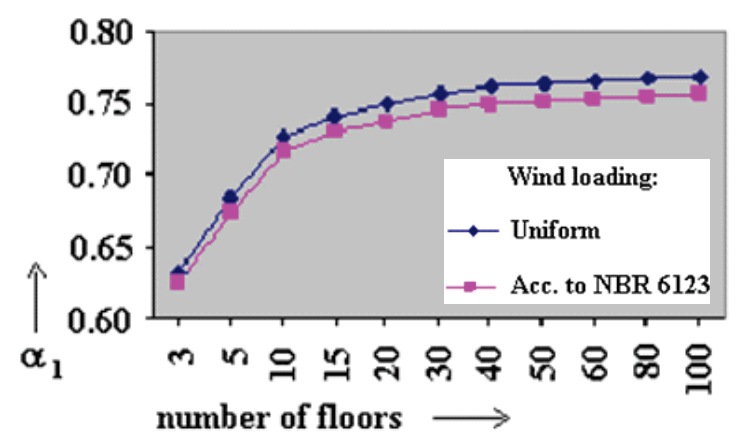

the closeness of the $\alpha_{1}$ values found for the two loading patterns. Considering that the roughness of category $V$ corresponds to the most far from uniform distribution loading pattern, other ground roughness categories were not tested, since it may be assumed that the resulting values would be intermediate between those obtained for the two aforementioned cases.

The good accuracy attained by the method proposed in the present study recommends its adoption in the derivation of formulas for the limit $\alpha_{1}$ of rigid-frame, wall-frame and core-frame bracing systems. Cases of unequally spaced floors and horizontal stiffness varying along the building height can also be considered. It must be emphasized that all of this has to be done in such a manner to keep the formulation simplicity, just one of the greater advantages of the instability parameter utilization.

Finally, it must be accentuated the need of adopting a more realistic analysis model for the tests: modeling of the structure as a three-dimensional frame, considering the floors as rigid diaphragms and including the shear deflections effect; effectuation of the nonlinear analysis through an incremental-iterative method; and a more accurate consideration of the physical nonlinearity, for example, by means of moment-curvature relations.

\section{References}

[01] Beck, H. and König, G.; Haltekräfte im Skeletbau. In: Beton- und Stahlbetonbau, $\mathrm{n}^{\circ} 62$, tome 1 (pp. 7-15) and tome 2 (pp. 37-42), Berlin, 1967.

[02] Vasconcelos, A.C.; Origem dos Parâmetros de Estabilidade $\alpha$ e $\gamma_{z}$. In: Revista IBRACON de Estruturas, $n^{\circ} 20$, pp. 56-60, São Paulo, 1998.

[03] Ellwanger, R.J.; A variable limit for the instability parameter of wall-frame or core-frame bracing structures. In: Revista IBRACON de Estruturas e Materiais, vol. 5, no 1, pp. 120-136, São Paulo, 2012.

[04] CEB - Comité Euro-International du Béton; CEB/FIP Manual of Buckling and Instability, The Construction Press, Lancaster, 1978.

[05] ABNT - Associação Brasileira de Normas Técnicas; NBR 6118 - Design of strucutral concrete Procedure, Rio de Janeiro, 2007. 
[06] Franco, M. and Vasconcelos, A.C.; Practical Assessment of Second Order Effects in Tall Buildings. In: Colóquio do CEB-FIP Model Code 1990, pp. 307-24, Rio de Janeiro, 1991.

[07] ABNT - Associação Brasileira de Normas Técnicas; NBR 6123 - Forças Devidas ao Vento em Edificações, Rio de Janeiro, 1987.

[08] Kantorovich, L.V. and Krylov, V.I.; Approximate Methods of Higher Analysis, New York, Interscience Publishers Inc., 1964.

[09] Smith, B.S. and Coull, A.: Tall Building Structures: Analysis and Design, New York, John Wiley \& Sons Inc., 1991. 